\title{
Preference between food and water as determined by hunger and thirst
}

JAMES ALLISON AND HAROLD LINDMAN

INDIANA UNIVERSITY

Implications of a theory relating preference between food and water to hunger and thirst were investigated by feeding, watering, or feeding and watering rats immediately before the preference test. Ss spent a greater proportion of time with food following prewatering than they did following prefeeding Preference for food following prefeeding and prewatering lay midway between the preference following prefeeding alone and the preference following prewatering alone. Both results were consistent with the theory.

The behavior of a hungry, thirsty rat confronted with a choice between food and water has potential significance for conflict theory, but early work with this paradigm (Young, 1945) did not exploit its theoretical possibilities. This paper places the paradigm in a theoretical context and reports a preliminary investigation of theoretical implications.

The general experimental setting is one in which $S$ is deprived of food and water prior to a $10 \mathrm{~min}$. test for preference between food and water. During the test period $S$ is allowed to shuttle between two adjacent goal boxes, one containing unlimited food, the other water. The dependent variable $P$ is the proportion of time $S$ spends in the food side rather than the water side.

The theory specifies that $P$ is determined by $F$, the probability that $\mathrm{S}$ will go from the water side to the food side during a given time interval, and $W$, the probability that $S$ will go from the food side to the water side during the same time interval. It is easy to show that asymptotically, $P=F /(F+W)$.

In principle, $F$ and $W$ can be varied by manipulating hours of food or water deprivation, but it is more practicable to manipulate amount eaten or drunk immediately before the test period. The theory assumes that feeding $S$ immediately before the test period (prefeeding) has a dual effect, decreasing $F$ and increasing $\mathrm{W}$ by a quantity $\alpha$. Psychologically, prefeeding decreases hunger. In the present context, prefeeding therefore decreases the tendency to go to the food side, and the tendency to stay in the food side. Similarly, it is assumed that watering S immediately before the test (prewatering) increases $F$ and decreases $W$ by a quantity $\beta$. Psychologically, prewatering decreases thirst, thereby decreasing the tendency to stay in the water side, and the tendency to go to the water side.

The theory is concerned with four distinct pretest conditions: $S$ is neither prefed nor prewatered $(0), S$ is prefed $(f), S$ is prewatered $(w)$, and $S$ is prefed and prewatered (fw). The theory states that

$$
\begin{aligned}
\mathbf{P}(0) & =\mathrm{F} /(\mathrm{F}+\mathrm{W}) \\
\mathbf{P}(f) & =(F-\alpha) /(F-\alpha+W+\alpha) \\
& =(F-\alpha) /(F+W) \\
\mathrm{P}(\mathrm{W}) & =(F+\beta) /(F+\beta+W-\beta) \\
& =(F+\beta) /(F+W) \\
\mathbf{P}(\mathbf{f w}) & =(F-\alpha+\beta) /(F-\alpha+\beta+W+\alpha-\beta) \\
& =(F-\alpha+\beta) /(F+W)
\end{aligned}
$$

From Equations (1) through (4),

$$
P(f)+P(w)-P(f w)=P(0)
$$

The remainder of the paper focuses on one obvious implication of the theory, and a second less obvious implication.

First, consider the case in which $S$ is either prefed (f) such that $\alpha>0$, or prewatered (w) such that $\beta>0$. Equations (2) and (3) clearly imply that under these conditions, $\mathrm{P}(\mathrm{w})>\mathrm{P}(\mathrm{f})$, i.e., a greater proportion of time will be spent on the food side following prewatering than following prefeeding.

Second, there may exist amounts of prefeeding and prewatering such that $\alpha=\beta$. If such amounts are employed, whether by chance or by design, the theory implies that $P(f w)=[P(f)+P(w)] / 2$. In other words, if such amounts are employed, preference for food following prefeeding and prewatering will lie midway between the preference following prefeeding alone and the preference following prewatering alone. This type of behavior will be referred to as averaging behavior. In the present context the occurrence of averaging behavior signifies that prefeeding and prewatering had equal, opposite effects upon $F$ and $W$.

The experiment reported below tested the first of these implications, and demonstrated incidentally the existence of averaging behavior.

\section{Method}

The apparatus was that described by Allison \& Silva (1965). It consisted of a U-shaped enclosure $7.5 \mathrm{in}$. high, with two $8 \times 12$ in. goal boxes forming the arms of the $U$, connected by a $4 \times 8$ in. alley forming the base. The enclosure had a plexiglas top covered with translucent waxed paper, wooden walls painted midgray, and a hardware-cloth floor covered with Plexiglas inserts. Each goal box contained a Plexiglas trough $6.25 \mathrm{in}$. wide, $2 \mathrm{in}$. high, and $2 \mathrm{in.} \mathrm{deep,} \mathrm{fastened}$ to the inside wall of the U. One trough was filled with $.045-\mathrm{gm}$ Noyes pellets, the other with tap water. A metal floor treadle pivoted at the center of the alley 
monitored S's crossings from one goal box to the other, and an event marker recorded time spent in each goal box.

The Ss were five adult male Long-Evans rats. For two weeks prior to testing the Ss were fed and watered $2 \mathrm{hr}$. daily, and for six days prior to testing were handled daily. They were habituated to the apparatus (with incentives present) immediately after feeding and watering during the three days before testing, spending 10 min. in each box on the first and second day, and exploring both boxes for $20 \mathrm{~min}$. on the third day. On any given day of the three day test period, at $22 \mathrm{hr}$. of food and water deprivation, $S$ consumed either $4 \mathrm{gm}$ of Noyes pellets (f), $1 \mathrm{ml}$ of water (w), or both (fw), immediately before the $10 \mathrm{~min}$. test. Each $\mathrm{S}$ was tested under all three conditions, and no two Ss received these conditions in the same order. Each $10 \mathrm{~min}$. test was begun by placing $\mathrm{S}$ on the treadle midway between the goal boxes, with its head oriented toward the food side.

\section{Results and Discussion}

All Ss spent more time in the food side than the water side under all three conditions. As the theory

Table 1. Proportion of Time Spent in Food Side

\begin{tabular}{cccc} 
S & Prefed & Prewatered & Prefed and prewatered \\
\hline 1 & .58 & .98 & .84 \\
2 & .67 & .89 & .70 \\
3 & .81 & .81 & .83 \\
4 & .78 & .82 & .80 \\
5 & .77 & .93 & .81 \\
Mean & .72 & .89 & .80 \\
\hline
\end{tabular}

predicts, Ss spent significantly more time in the food side following prewatering than they did following prefeeding ( $p=.03$ by the randomization test).

Individual data and condition means are presented in Table 1. The means show that the amounts of prefeeding and prewatering used in this experiment produced averaging behavior. That is, averaging behavior is said to occur if $P(f w)=[P(f)+P(w)] / 2$, and .80 is very nearly equal to $(.72+.89) / 2$.

According to the theory, averaging behavior need not occur. For example, suppose $\mathrm{X}$ and $\mathrm{Y}$ are amounts of prefeeding and prewatering respectively which produce averaging behavior. The theory implies that $P(f w)$ will be greater than the average of $P(f)$ and $P(w)$ if $\alpha$ is decreased ( $S$ is prefed less than amount $X$ ) or if $\beta$ is increased ( $S$ is prewatered more than amount $Y)$. Similarly, it can be shown that $P(f w)$ will be less than the average of $P(f)$ and $P(w)$ if $\alpha$ is increased ( $S$ is prefed more than amount $X$ ) or if $\beta$ is decreased ( $S$ is prewatered less than amount $Y$ ). The theory seems highly vulnerable. As Equation (5) indicates, the theory can be examined even more critically by testing Ss under all four conditions, rather than the three which suffice to investigate averaging or departures from averaging behavior.

\section{References}

Allison, J., \& Silva, Maria R. Time spent with food and nonfood incentives as a function of food deprivation. Psychon. Sci., $1965,2,63-64$.

Young, P. T. Studies of food preference, appetite and dietary habit: IV. The balance between hunger and thirst. J. comp. Psychol., $1945,38,135-174$. 